\title{
Comparing Attitudes to Containment Measures of Patients, Health Care Professionals and Next of Kin
}

\author{
Thomas Reisch ${ }^{1,2 \star}$, Simone Beeri ${ }^{1}$, Georges Klein ${ }^{3}$, Philipp Meier ${ }^{1}$, Philippe Pfeifer ${ }^{1,2}$, \\ Etienne Buehler ${ }^{4}$, Florian Hotzy ${ }^{4}$ and Matthias Jaeger ${ }^{4}$ \\ ${ }^{1}$ Hospital of Psychiatry Muensingen, Bern, Switzerland, ${ }^{2}$ University Hospital of Psychiatry and Psychotherapy, Bern, \\ Switzerland, ${ }^{3}$ Département de Psychiatrie et Psychothérapie du Centre Hospitalier du Valais Romand, Monthey, Switzerland, \\ ${ }^{4}$ Department for Psychiatry, Psychotherapy and Psychosomatics, University Hospital of Psychiatry Zurich, Zurich, Switzerland
}

Background: In clinical psychiatric practice, health care professionals (HCP) must decide in exceptional circumstances after the weighing of interests, which, if any, containment measures including coercion are to be used. Here, the risk for patients, staff, and third parties, in addition to therapeutic considerations, factor into the decision. Patients' preference and the inclusion of relatives in these decisions are important; therefore, an understanding of how patients and next of kin (NOK) experience different coercive measures is crucial for clinical decision making. The aim of this study is to compare how patients, HCP, and NOK assess commonly used coercive measures.

Methods: A sample of 435 patients, $372 \mathrm{HCP}$, and 230 NOK completed the Attitudes to Containment Measures Questionnaire (ACMQ). This standardized self-rating questionnaire assessed the degree of acceptance or rejection of 11 coercive measures.

ZfP Südwürttemberg, Germany

Reviewed by:

Charles Bonsack

Lausanne University Hospital (CHUV),

Switzerland

Chantal Ski

Queen's University Belfast,

United Kingdom

${ }^{*}$ Correspondence:

Thomas Reisch

thomas.reisch@pzmag.ch

Specialty section

This article was submitted to

Public Mental Health,

a section of the journal

Frontiers in Psychiatry

Received: 25 May 2018 Accepted: 05 October 2018

Published: 26 October 2018

Citation:

Reisch T, Beeri S, Klein G, Meier P.

Pfeifer P, Buehler E, Hotzy F and Jaeger M (2018) Comparing Attitudes to Containment Measures of Patients, Health Care Professionals and Next of

Kin. Front. Psychiatry 9:529.

doi: 10.3389/fpsyt.2018.00529
Results: In general, HCPs rated the coercive measures as more acceptable than did NOK and patients. The largest discrepancy in the ratings was found in regard to the application of coercive intramuscular injection of medication (effect size: 1.0 HCP vs. patients). However, the ratings by NOK were significantly closer to the patients' ratings compared to patients and HCP. The only exception was the acceptance of treatment in a closed acute psychiatric ward, which was deemed significantly more acceptable by NOK than by patients. Also, patients who had experienced coercive measures themselves more strongly refused other measures.

Conclusion: Patients most firmly rejected intramuscular injections, and the authors agree that these should only be used with reservation considering a high threshold. This knowledge about the discrepancy of the ratings should therefore be incorporated into professional training of HCP.

Keywords: containment measures, coercive measures, coercion, fixation, intramuscular injection, mechanical restraint, physical

\section{INTRODUCTION}

The implementation of coercive measures presents a major challenge for health care professionals (HCP). HCP face the dilemma of being responsible for safety while at the same time being obligated to promote therapy and take into account the self-determination and free will of the patient (1). Conversely, patients experience coercive measures as a "distinct negative incident" (2) 
and frequently as a traumatizing one (3). Coercive measures are usually applied to avert destructive actions against oneself, other patients, or staff. Aggression, especially assault of third persons, disorganization, and agitation, are common catalysts for coercive measures (4-7). Moreover, HCP often see the therapeutic effects of coercive measures $(2,8)$. Overall, the literature shows that some HCP see coercive measures as a necessary "emergency break" (9). However, several studies have demonstrated the negative effects of coercive measures, which have an unfavorable impact on the therapeutic relationship (10-13). In general, coercive measures lead to lower treatment satisfaction (14), reduce the effectiveness of the therapy (15), and prolong the duration of inpatient treatment (16). Still, it must be assumed that this is at least partly due to the fact that patients, especially those who experience coercive measures, often suffer from serious mental illness (17).

Coercive measures are exercised on a significant number of patients with strong differences between and within countries (18-20). In many countries, coercive measures are applied to $10-20 \%$ of all psychiatric inpatients (21-23). Significantly higher rates are reported in samples from other countries (19). In China, for example, $51.3 \%$ of all inpatients experienced coercive measures during their treatment; however, it must be noted that these international differences are related to variance in national legislation (20). Nonetheless, culture-specific attitudes and therapeutic approaches may also play a role in these major differences. In addition to differences in the absolute frequency of implementation between countries, the type of coercive measure applied also varies $(23,24)$. For example, in Germany, patients are more likely to be subject to mechanical restraint (23), a measure that is rarely used in English-speaking countries (25). In these countries, it is more common to physically restrain patients (26). Some countries, such as Switzerland (27) or the Netherlands (23), have high rates of seclusion.

Generally, a differentiation can be made between more and less invasive coercive measures. Measures, such as PRN (pro re nata) medication, observation, and time-out, are considered less invasive and are therefore preferable to the more invasive measures (28-30). However, particularly with violent patients, measures that more strongly limit personal freedom, including physical restraints, seclusion, and forced medication, may become inevitable (31).

Yet, the decision as to which of these more invasive measures is used is not rationally derivable but subject to the traditions of psychiatric clinics as well as legislation $(22,32)$. Researchers have pointed out that the patient's preference, often dependent on their previous experience with coercive measures, should be considered (33). Perceived coercion is an important mediating factor in the acceptance of coercive measures and is therefore, indirectly, responsible for the treatment outcome (34). When a higher level of coercion is perceived, patients feel powerless and inferior, and HCP suffer more guilt (35). These diverse perspectives provide context as to why the individual measures are perceived differently in various groups. Different perceptions of patients and HCP have been described in studies, especially in regard to forced intramuscular medication. While HCP were found to be more in favor of this measure, patients strongly rejected it $(33,36)$. Thus, it could be shown that patients who have experienced forced medication (orally or intramuscularly administered) also evaluated the treatment negatively 3 months later (disapproval of treatment) (37). A greater frequency of using forced medication also correlated with an increased negative evaluation of coercive measures (38). Differing ratings are also a factor in other forms of coercive measures. For example, patients have a significantly more negative assessment of the closed door of psychiatric wards than do HCP (39).

The involvement of NOK concerning the decision for coercive measures is considered standard today (40) (SAMW guideline). Research on ratings of coercive measures, however, is nearly nonexistent. Ranieri et al. (41) showed that involuntary admission is perceived as less restrictive by NOK than by patients. This suggests that differences between NOK and patients in the ratings of specific coercive measures are to be expected.

In summary, there are varying perspectives, roles, and emotions of patients, HCP, and NOK regarding containment, especially coercive measures $(2,42-44)$. The acknowledgment of these differing attitudes is important for the therapeutic relationship and thus the treatment. The aim of this study is to highlight this very area of conflict, and the knowledge gained will be used to develop a better understanding to improve dialogue with patients $(45,46)$ and the training of HCP $(44)$. Over time, such improvements could help reduce the stigma of psychiatry (10) and psychiatric clinics as safeguarding institutions (47).

Based on the cited literature and the previously mentioned considerations, we expect that $\mathrm{HCP}$ will generally show a higher acceptance of all coercive measures. Patients, however, will be more likely to reject coercive measures. We also expect that NOK will reject coercive measures less often than patients but more often than the HCP. In addition, we expect that the 3 samples will differ widely in their attitudes toward forced medication in particular. Patients who have experienced such a measure should, according to Dack, Ross, and Bowers (38), also evaluate other highly coercive measures more negatively.

\section{METHOD}

\section{Data Collection, In- and Exclusion Criteria, Ethics, and Anonymization}

The study was conducted with 3 samples (patients, HCP, NOK) at 3 Swiss sites, i.e., the University Hospital of Psychiatry Zurich (Canton of Zurich), the Psychiatric Hospital Malévoz (Monthey, Canton of Valais), and the Hospital of Psychiatry Münsingen (Canton of Bern). The study included all patients with sufficient verbal communication necessary to understand the questionnaire and give informed consent.

A study nurse instructed the patients how to complete the questionnaire. The anonymization of the patient questionnaires took place after entering the data. HCP (mental health nurses, physicians, and psychologists) completed the questionnaire anonymously during working hours. NOK were contacted by mail, or directly if one of their relatives was hospitalized in one of the 3 clinics during the study period. The NOK questionnaire was sent to NOK and additionally asked for age and relationship 
to the treated relative. Due to the anonymization, a direct connection of NOK questionnaires to patient questionnaires was not possible.

The cantonal Ethics Commission Bern (Ref.-nr. KEK-BE: 2015-00074) reviewed and approved the study. This approval was binding for all survey sites.

\section{Sample}

The study was carried out among patients, NOK, and HCP on psychiatric acute wards of the 3 psychiatric hospitals mentioned above using unselected samples. Overall, data from 1,037 study participants was included. A minority of the participating patients was compulsory admits (20.6\%).

Of the NOK, $38.2 \%$ were parents of the patients $(N=84)$, 9.5\% were children of the patients $(N=21), 13.2 \%$ were siblings $(N=29), 22.3 \%$ partners $(N=49), 16.8 \%$ other related persons $(N=37)$, and 10 were missing this specification. The HCP group consisted of $66.4 \%$ nurses $(N=243), 25.1 \%$ physicians $(N=92)$, and $8.5 \%$ psychologists $(N=31)$. There were $6 \mathrm{HCP}$ responses that lacked specific occupational data. For more details, see Table 1.

\section{ACMQ}

The Attitudes to Containment Measures Questionnaire (ACMQ) is a self-rating paper-and-pencil questionnaire that has been validated (38) and used in several publications from different countries, and thus from different cultures $(24,25,28,30,39,48-$ 55). One disadvantage of the ACMQ is that it also collects data on coercive measures that are uncommon or not used at all in Switzerland, such as the net bed.

The 11 main items of the ACMQ have a uniform structure. The specific coercive measure is briefly described and illustrated by a picture, then the participant of the study is asked how acceptable the measure is on a 5-point Likert scale [strongly agree (0) to strongly disagree (5)]. A high value means a high rejection or, respectively, a low acceptance. For each item, the patients were also asked whether they had already experienced this measure. HCP study participants were asked if they had already executed the specific containment measure. In NOK, we inquired as to whether this measure had been administered to their kin. The ACMQ encompasses the following coercive measures: PRN medication, physical restraint, intermittent observation, seclusion, time-out, compulsory intramuscular medication, psychiatric intensive care, mechanical restraint, constant observation, net bed, and open area seclusion.

\section{Statistical Analyses}

The statistical analysis was done using SPSS Version 24. Statistical analyses were carried out using standard procedures. Arithmetic means of items were compared using $t$-test for independent samples. The test results were checked for multiple testing by Bonferroni corrections, and the quantification of the differences was determined by effect sizes. In this connection, the pooled standard deviations of the respective group results were taken into account. Due to missing data, there were minor deviations of the number of questionnaires in individual analyses.

Regarding patients, it was distinguished whether they had experienced a coercive measure themselves. Furthermore, the results of compulsory admitted patients were compared to patients treated on a voluntary basis. Whether a patient had never experienced or had experienced at least one of the highly restrictive coercive measures in the past would cause differences in their rating of the measures.

\section{RESULTS}

\section{Comparing the Results of Patients, HCP and NOK}

The group analysis showed a notable trend. The degree of rejection of all measures was higher among patients than NOK, and higher among NOK than HCP. The general pattern of which coercive measure was rejected the most did not differ between the three groups. All groups rejected the net bed the most, all groups ranked mechanical restraints as the second most unfavorable measure, with seclusion as the third. The biggest difference with respect to the ranking was seen in regards to compulsory intramuscular medication. While it was ranked as the fourth most unfavorable measure by patients and NOK, it was ranked eighth by HCP.

This result was confirmed by analysis of the quantitative differences in the assessments of the individual measures between the groups. Compulsory intramuscular medication produced the largest effect size and thus the largest differences in direct comparison between HCP and patients and between HCP and NOK. The comparison between NOK and HCP also shows that seclusion and mechanical restraint are rated differently. Acceptance of treatment on a locked acute ward is the only measure that shows no significant difference between HCP and NOK. The differences between NOK and patients are small on average: A medium effect size was found only for treatment on

TABLE 1 | Sample.

\begin{tabular}{|c|c|c|c|c|c|c|c|}
\hline & $N$ (total) & $N(\mathrm{Mu})$ & $N$ (Mo) & $N(\mathrm{Zu})$ & Age (ys) & SD & Female (\%) \\
\hline Patients & 435 & 97 & 236 & 102 & 40.7 & 13.3 & 46.1 \\
\hline $\mathrm{HCP}$ & 372 & 146 & 114 & 112 & 37.6 & 11.7 & 60.4 \\
\hline NOK & 230 & 99 & 63 & 68 & 49.3 & 16.1 & 58.4 \\
\hline Total & 1,037 & 342 & 413 & 282 & 42.0 & 14.1 & 53.9 \\
\hline
\end{tabular}

Mu, Psychiatric Hospital of Muensingen; Mo, Psychiatric Hospital of Monthey; Zu, University Hospital of Psychiatry Zurich; HCP, health care professionals; NOK, next of kin. 
an acute closed ward, as NOK rated this measure as significantly more acceptable (see Table 2; Figure 1).

\section{Subanalyses of the Patient Sample}

Patients admitted on a compulsory basis tended to rate coercive measures as less acceptable than voluntarily hospitalized patients. The strongest effect sizes were found for physical restraint and compulsory intramuscular medication (see Table 3).

Patients who experienced at least one strongly restricting coercive measure (physical restraint, seclusion, compulsory intramuscular medication or mechanical restraint; $N=38$, $34.7 \%)$ rated the coercive measures as less acceptable compared to patients who had not experienced coercion $(t=3.15$, $p=0.002$ ). The effect size (ES) of this difference was 0.33 . Significantly higher rejections were found for PRN medication $(t=2.29, p=0.023$, ES 0.26.), physical restraint $(t=3.14$, $p=0.002$, ES 0.32), compulsory intramuscular medication $(t=2.89, p=0.004$, E. 0.31$)$, mechanical restraint $(t=2.10$, $p=0.037$, ES 0.22$)$ and the network bed $(t=2.36, p=0.019$, ES $=0.25)$. After the Bonferroni correction, only physical restraint and coercive medication were statistically significant. If this analysis is limited in line with Dack et al. (2) to patients who had experienced a compulsory intramuscular medication, a virtually identical result is obtained (mean value of all measures $t=2.98$, $p=0.003, \mathrm{ES}=0.35$ ).

\section{DISCUSSION}

According to our hypothesis, patients and NOK consistently rejected all coercive measures more strongly than HCP. The latter presumably consider the potential benefits of these measures more often and feel responsible for preventing harm to other patients and themselves. The low values for HCP may also be seen as a justification for their own behavior.

When viewing the ranking of the ratings over the absolute assessment values of the measures, all three study groups show an identical ranking order for the three items with the highest rating. In line with several publications (29, 30, 54), Swiss patients, NOK, and HCP most clearly rejected the net bed. This measure is not applied in Switzerland and is likely, as in Finland, perceived as "inhumane and cruel" (28). Mechanical restraints and seclusion were rejected second- and third-most by all groups. In contrast to the net bed, these measures are widely used in German-speaking countries. Patients preferred pro re nata medication, physical restraint, psychiatric intensive care and constant observation to compulsive intramuscular injection. In some situations, intramuscular medication may be difficult to avoid. However, in respect to the results of our study, clinicians should evaluate whether less aversive measures, such as pro re nata medication, psychiatric intensive care and constant observation can be used in its place. In other cases, injection might be prevented by steps, such as changing the culture or atmosphere of the ward. Notably, compulsive intramuscular injection was preferred by the patients to mechanical restraint. These two measures are often combined in clinical practice. Clinicians should in these cases also evaluate whether mechanical

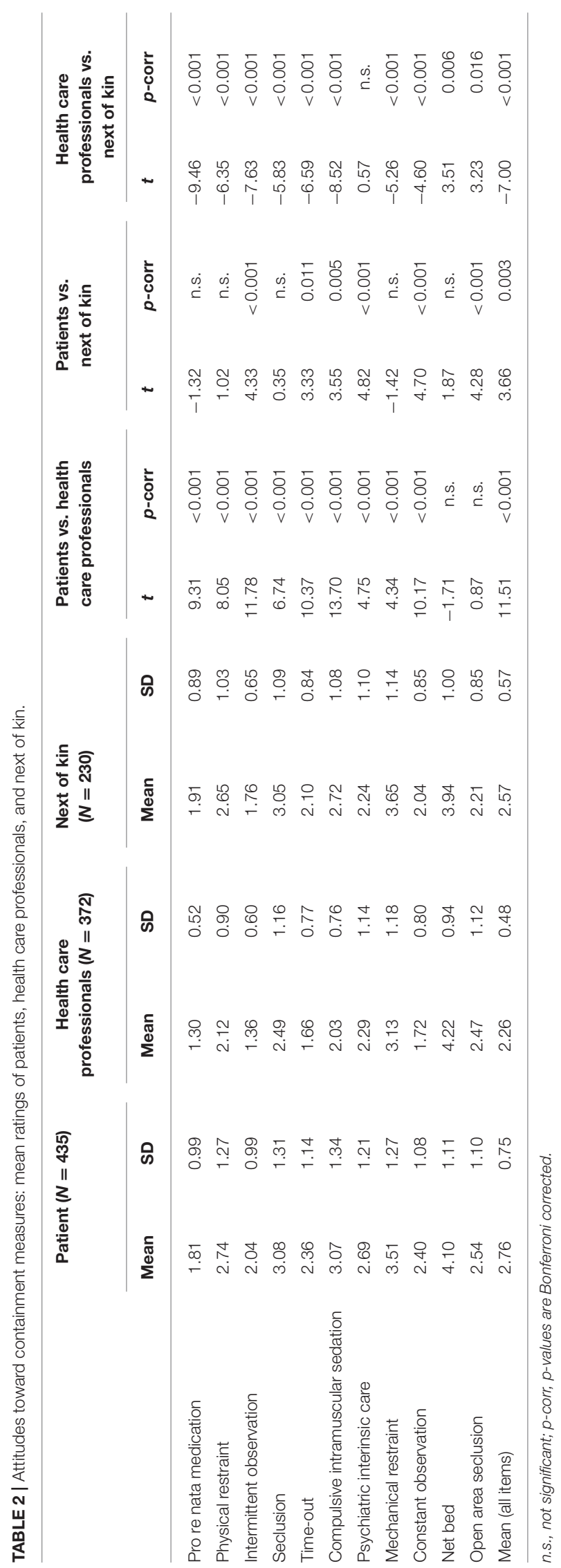




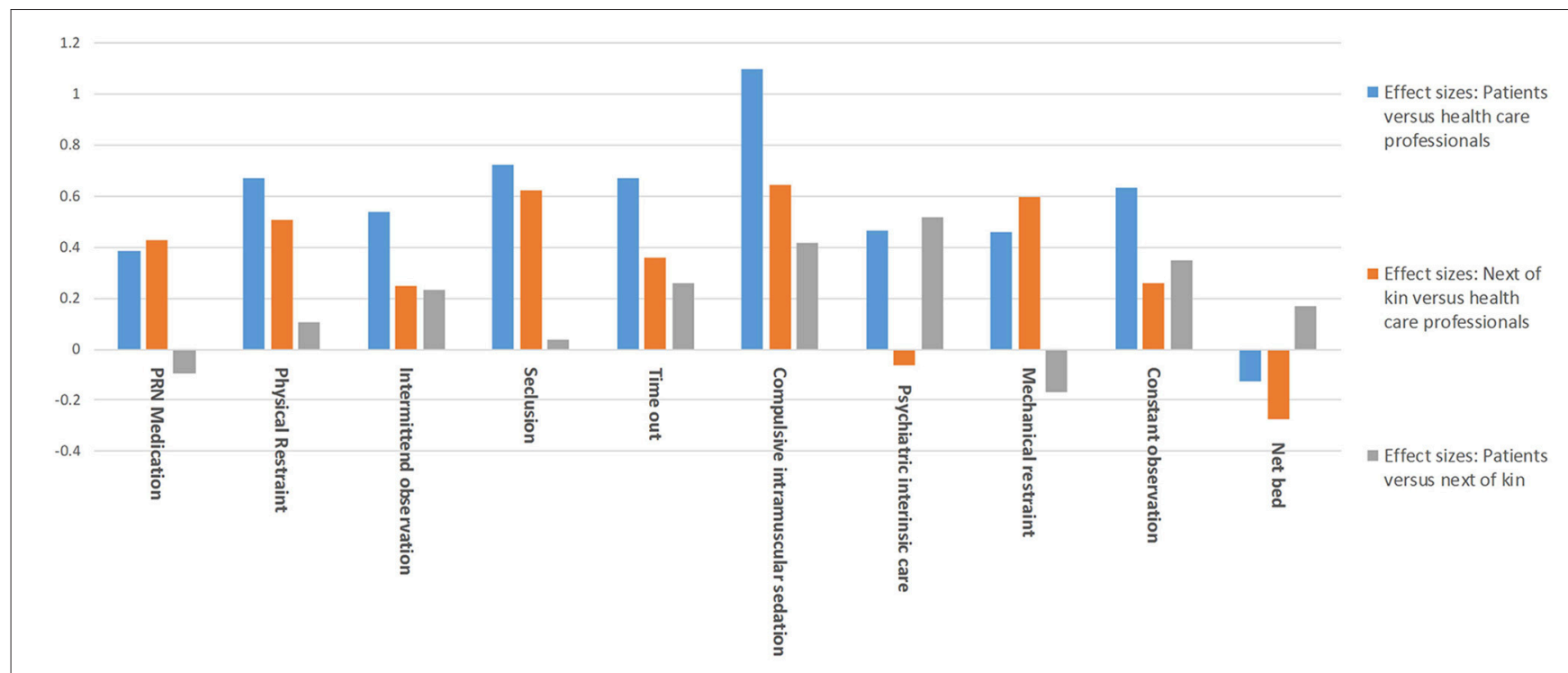

FIGURE 1 | Effect sizes: comparing ratings of patients, health care professionals, and next of kin.

TABLE 3 | Comparing ACMQ ratings of voluntary vs. compulsorily admitted patients.

\begin{tabular}{|c|c|c|c|c|c|c|c|c|}
\hline & \multicolumn{2}{|c|}{ Voluntary admitted $(N=352,81.1 \%)$} & \multicolumn{2}{|c|}{ Complusorily admitted ( $N=82,18.9 \%)$} & \multicolumn{4}{|c|}{ Statistics } \\
\hline & Mean & SD & Mean & SD & $t$ & $p$ & $p$-corr & ES \\
\hline Pro re nata medication & 1.74 & 0.94 & 2.14 & 1.12 & -2.97 & 0.004 & 0.044 & 0.39 \\
\hline Physical restraint & 2.61 & 1.21 & 3.32 & 1.38 & -4.28 & $<0.001$ & $<0.001$ & 0.55 \\
\hline Intermittent observation & 1.99 & 0.95 & 2.25 & 1.15 & -1.89 & n.s. & n.s. & 0.25 \\
\hline Seclusion & 3.07 & 1.31 & 3.15 & 1.33 & -0.50 & n.s. & n.s. & 0.06 \\
\hline Time-out & 2.31 & 1.11 & 2.59 & 1.24 & -1.97 & 0.049 & n.s. & 0.23 \\
\hline Compulsive intramuscular sedation & 2.95 & 1.31 & 3.57 & 1.35 & -3.77 & $<0.001$ & 0.002 & 0.46 \\
\hline Psychiatric interinsic care & 2.67 & 1.18 & 2.78 & 1.29 & -0.70 & n.s. & n.s. & 0.08 \\
\hline Mechanical restraint & 3.43 & 1.27 & 3.86 & 1.20 & -2.79 & 0.006 & n.s. & 0.35 \\
\hline Constant observation & 2.34 & 1.02 & 2.68 & 1.26 & -2.23 & 0.028 & n.s. & 0.29 \\
\hline Net bed & 4.04 & 1.14 & 4.34 & 0.98 & -2.20 & 0.028 & n.s. & 0.28 \\
\hline Open area seclusion & 2.45 & 1.03 & 2.90 & 1.27 & -2.99 & 0.003 & 0.042 & 0.39 \\
\hline Mean (all items) & 2.69 & 0.73 & 3.06 & 0.78 & -4.08 & $<0.001$ & 0.001 & 0.49 \\
\hline
\end{tabular}

ACMQ, Attitudes to Containment Measures Questionnaire; n.s., not significant; p-corr, p-values are Bonferroni corrected.

restraint can be at least avoided by applying compulsory injection only.

The largest rating differences between HCP and patients surround compulsory intramuscular injection of medication. With an effect size $>1$, this difference may be related to the conviction of HCP that intramuscular injection of medication is therapeutically necessary (5). The focus of HCP on applying treatment interventions rather than mere security measures explains these beliefs. Still, there is a risk that HCP use these measures with a relatively low threshold and thus insufficiently consider their negative effects, namely the deterioration of the therapeutic relationship due to disapproval of coercion by patients (37). Additionally, the therapeutic effect of a compulsory medication could not be verified by evidence (26), and that medication is frequently used for temporary control of behavior (3).

In accordance with Dack et al. (38), we observed that patients who were medicated against their will had more negative attitudes toward all coercive measures. The differing ratings of the patients, as well as the ratings of all measures taken together, show that such an act has high costs. Therefore, HCP must avoid this intervention whenever possible.

More than a third of all coercive measures are triggered by a HCP-patient interaction (47). Consequently, the use of compulsory medication is preventable in advance. Solutions may include greater sensitivity to the use of informal coercion, which may represent a precursor of coercive measures and lead to disruptions in the therapeutic relationship (56). Reducing the 
consequences of coercive measures could include debriefing of events, which in Switzerland is considered standard (40) (SAMW guideline). Clinicians should actively seek patient perspectives on compulsory medication retroactively to minimize secondary negative impact. The reduction of perceived coercion should always be an objective while administering coercive measures to reduce negative effects within the therapeutic relationship. Clinicians can achieve this through transparent communication, choices concerning coercive measures, sound justification for these measures, and respect for the patients' perspectives $(31,57)$.

Patients who were admitted compulsorily were more likely to show a negative attitude toward coercive measures, which concurs with numerous publications $(5,12)$. The main reason for these negative attitudes may be the acute illness of compulsory patients at the beginning of their treatment (19). Unfortunately, our results could not differentiate whether patients had a negative attitude before beginning their treatment, if a negative stance to psychiatric treatment causes their negative attitude, or if they acquired their attitude after experiencing involuntary admission.

In general, NOK reject coercive measures significantly less strongly than do patients. This result is consistent with Ranieri et al. (41). This likely stems from NOK approval toward coercive treatment conducted for the wellbeing of their relatives. They often find themselves caught in an ambivalent position, as they simultaneously want to avoid patient suffering from restricted autonomy and freedom of movement. In absolute terms, however, NOK displayed ratings much closer to those of patients. It is noteworthy that the treatment on the locked acute

\section{REFERENCES}

1. Voskes Y, Evenblij K, Noorthoorn E, Porz R, Widdershoven G. Moral case deliberation about coercion in psychiatry. Dilemmas, value and implementation. Psychiatr Pract. (2014) 41:364-70. doi: 10.1055/s-0034-1370292

2. Van Der Merwe M, Muir-Cochrane E, Jones J, Tziggili M, Bowers L. Improving seclusion practice: implications of a review of staff and patient views. J Psychiatr Ment Health Nurs. (2013) 20:203-15. doi: 10.1111/j.1365-2850.2012.01903.x

3. Kinner SA, Harvey C, Hamilton B, Brophy L, Roper C, McSherry B, et al. Attitudes towards seclusion and restraint in mental health settings: findings from a large, community-based survey of consumers, carers and mental health professionals. Epidemiol Psychiatr Sci. (2017) 26:535-44. doi: $10.1017 /$ s2045796016000585

4. Flammer E, Steinert T. Involuntary medication, seclusion, and restraint in German psychiatric hospitals after the adoption of legislation in 2013. Front Psychiatry (2015) 6:153. doi: 10.3389/fpsyt.2015. 00153

5. Jarrett M, Bowers L, Simpson A. Coerced medication in psychiatric inpatient care: literature review. J Adv Nurs. (2008) 64:538-48. doi: 10.1111/j.1365-2648.2008.04832.x

6. Keski-Valkama A, Sailas E, Eronen M, Koivisto AM, Lonnqvist J, KaltialaHeino R. The reasons for using restraint and seclusion in psychiatric inpatient care: a nationwide 15-year study. Nordic J Psychiatr. (2010) 64:136-44. doi: 10.3109/08039480903274449

7. Larue C, Dumais A, Drapeau A, Menard G, Goulet MH. Nursing practices recorded in reports of episodes of seclusion. Iss Ment Health Nurs. (2010) 31:785-92. doi: 10.3109/01612840.2010.520102

8. Molewijk B, Kok A, Husum T, Pedersen R, Aasland O. Staff's normative attitudes towards coercion: the role of moral doubt and professional ward is the only item that did not differ between NOK and HCP while also showing a significant difference between NOK and patients. Taken together, although in favor of treatment on the acute ward, NOK are critical of the concrete measures. In other words, they agree that their relatives must be treated, but not on the methods of treatment. Considering the essential role of NOK in patient care, especially of seriously ill psychiatric patients, it is vital to actively include NOK after administering coercive measures (58).

In summary, we can conclude that our study has several limitations and strengths. One such shortcoming is the relatively low participation rates of NOK and patients. In particular, patients treated with coercive measures often refused participation in the study. Further, data collection was done after stabilization; however, it did occur during treatment on acute wards. It is possible that residual symptoms and the patient's own experiences influenced the results. The main strength of our study is, according to our literature review, that it is the first to examine ratings of containment measures by NOK, HCP, and patients comparatively. In addition, the large sample allows for high statistical power and the detection of medium effects.

\section{AUTHOR CONTRIBUTIONS}

PM: translation, editing, and writing; SB: research, data collection, and writing; TR and MJ: research design, data analysis, and writing; GK and PP: data collection, writing, and analysis; EB and $\mathrm{FH}$ : data collection and analysis. context-a cross-sectional survey study. BMC Med Ethics (2017) 18:37. doi: 10.1186/s12910-017-0190-0

9. Sibitz I, Scheutz A, Lakeman R, Wardrobe B, Schaffer M, Amering M. Impact of coercive measures on life stories: qualitative study. Br J Psychiatry (2011) 199:239-44. doi: 10.1192/bjp.bp.110.087841

10. Hotzy F, Jaeger M. Clinical relevance of informal coercion in psychiatric treatment-a systematic review. Front Psychiatry (2016) 7:197. doi: 10.3389/fpsyt.2016.00197

11. Rugkasa J, Canvin K, Sinclair J, Sulman A, Burns T. Trust, deals and authority: community mental health professionals' experiences of influencing reluctant patients. Community Ment Health J. (2014) 50:886-95. doi: 10.1007/s10597-014-9720-0

12. Sheehan KA, Burns T. Perceived coercion and the therapeutic relationship: a neglected association? Psychiatr Serv. (2011) 62:471-6. doi: 10.1176/appi.ps.62.5.471

13. Theodoridou A, Schlatter F, Ajdacic V, Rossler W, Jager M. Therapeutic relationship in the context of perceived coercion in a psychiatric population. Psychiatr Res. (2012) 200:939-44. doi: 10.1016/j.psychres.2012.04.012

14. McNiel DE, Gormley B, Binder RL. Leverage, the treatment relationship, and treatment participation. Psychiatr Serv. (2013) 64:431-6. doi: 10.1176/appi.ps.201200368

15. Van Dorn RA, Swartz M, Elbogen EB, Swanson JW. Perceived fairness and effectiveness of leveraged community treatment among public mental health consumers in five US cities. Int J Forensic Ment Health (2005) 4:119-33. doi: 10.1080/14999013.2005.10471218

16. Knutzen M, Bjorkly S, Eidhammer G, Lorentzen S, Mjosund NH, Opjordsmoen S, et al. Characteristics of patients in pharmacological and restraint-a register study in three Norwegian acute psychiatric wards. Psychiatr Res. (2014) 215:127-33. doi: 10.1016/j.psychres.2013.10.024

17. Knutzen M, Mjosund NH, Eidhammer G, Lorentzen S, Opjordsmoen S, Sandvik L, et al. Characteristics of psychiatric inpatients who experienced 
restraint and who did not: a case-control study. Psychiatr Serv. (2011) 62:4927. doi: $10.1176 /$ ps.62.5.pss6205_0492

18. Kalisova L, Raboch J, Nawka A, Sampogna G, Cihal L, Kallert TW, et al. Do patient and condition-related characteristics influence the use of coercive measures? Results from the EUNOMIA international study. Soc Psychiatr Psychiatr Epidemiol. (2014) 49:1619-29. doi: 10.1007/s00127-0140872-6

19. Raboch J, Kališová L, Nawka A, Kitzlerova E, Onchev G, Karastergiou A, et al. Use of coercive measures during involuntary hospitalization: findings from ten European countries. Psychiatr Serv. (2010) 61:1012-7. doi: 10.1176/appi.ps.61.10.1012

20. Steinert T, Lepping P, Bernhardsgrutter R, Conca A, Hatling T, Janssen W, et al. Incidence of seclusion and restraint in psychiatric hospitals: a literature review and survey of international trends. Soc Psychiatr Psychiatr Epidemiol. (2010) 45:889-97. doi: 10.1007/s00127-009-0132-3

21. Bilanakis N, Kalampokis G, Christou K, Peritogiannis V. Use of coercive physical measures in a psychiatric ward of a general hospital in Greece. Int J Soc Psychiatr. (2010) 56:402-11. doi: 10.1177/002076400 9106620

22. Hendryx M, Trusevich Y, Coyle F, Short R, Roll J. The distribution and frequency of seclusion and/or restraint among psychiatric inpatients. J Behav Health Serv Res. (2010) 37:272-81. doi: 10.1007/s11414-009-9191-1

23. Noorthoorn E, Lepping P, Janssen W, Hoogendoorn A, Nijman H, Widdershoven G, et al. One-year incidence and prevalence of seclusion: Dutch findings in perspective internationally. Soc Psychiatr Psychiatr Epidemiol. (2015) 50:1857-69. doi: 10.1007/s00127-015-1094-2

24. Bowers L, van der Werf B, Vokkolainen A, Muir-Cochrane E, Allan T, Alexander J. International variation in containment measures for disturbed psychiatric inpatients: a comparative questionnaire survey. Int J Nurs Stud. (2007) 44:357-64. doi: 10.1016/j.ijnurstu.2006.01.005

25. Bowers L, Van der Merwe M, Paterson B, Stewart D. Manual restraint and shows of force: the City-128 study. Int J Ment Health Nurs. (2012) 21:30-40. doi: $10.1111 / j .1447-0349.2011 .00756 . x$

26. Steinert T. After 200 years of psychiatry: are mechanical restraints in Germany inevitably barred? Psychiatr Pract. (2011) 38:348-51. doi: 10.1055/s-0031-1276871

27. Martin V, Kuster W, Baur M, Bohnet U, Hermelink G, Knopp M, et al. Incidence of coercive measures as an indicator of quality in psychiatric hospitals. Problems of data recording and processing, preliminary results of a benchmarking study. Psychiatr Pract. (2007) 34:26-33. doi: 10.1055/s-2005-866920

28. Hottinen A, Valimaki M, Sailas E, Putkonen H, Joffe G, Noda T, et al. Underlying patients' mental illness: a questionnaire survey in Finnish adolescent psychiatry. J Child Adolescent Psychiatr Nurs. (2012) 25:219-23. doi: $10.1111 /$ jcap. 12006

29. Ozcan NK, Bilgin H, Badirgali Boyacioglu NE, Kaya F. Student nurses' attitudes towards professional containment methods used in psychiatric wards and perceptions of aggression. Int J Nurs Pract. (2014) 20:346-52. doi: 10.1111/ijn.12157

30. Whittington R, Bowers L, Nolan P, Simpson A, Neil L. Approval ratings of inpatient coercive interventions in a national sample of mental health service users and staff in England. Psychiatr Serv. (2009) 60:792-8. doi: 10.1176/appi.ps.60.6.792

31. Cowman S, Bjorkdahl A, Clarke E, Gethin G, Maguire J. A descriptive survey study of violence management and priorities among psychiatric staff in mental health services, across seventeen European countries. BMC Health Serv Res. (2017) 17:59. doi: 10.1186/s12913-017-1988-7

32. Bowers L, Alexander J, Simpson A, Ryan C, Carr-Walker P. Cultures of psychiatry and the professional socialization process: the case of containment methods for disturbed patients. Nurse Edu Today (2004) 24:435-42. doi: 10.1016/j.nedt.2004.04.008

33. Georgieva I, Mulder CL, Wierdsma A. Patients' preference and experiences of forced medication and seclusion. Psychiatr Q. (2012) 83:1-13. doi: 10.1007/s11126-011-9178-y

34. Bindman J, Reid Y, Szmukler G, Tiller J, Thornicroft G, Leese M. Perceived coercion at admission to psychiatric hospital and engagement with followup-a cohort study. Soc Psychiatr Psychiatr Epidemiol. (2005) 40:160-6. doi: 10.1007/s00127-005-0861-x
35. Larsen IB, Terkelsen TB. Coercion in a locked psychiatric ward: perspectives of patients and staff. Nurs Ethics (2014) 21:426-36. doi: $10.1177 / 0969733013503601$

36. Haglund K, Von Knorring L, Von Essen L. Forced medication in psychiatric care: patient experiences and nurse perceptions. J Psychiatr Ment Health Nurs. (2003) 10:65-72. doi: 10.1046/j.1365-2850.2003.00555.x

37. McLaughlin P, Giacco D, Priebe S. Use of coercive measures during involuntary psychiatric admission and treatment outcomes: data from a prospective study across 10 European countries. PLoS ONE (2016) 11:e0168720. doi: 10.1371/journal.pone.0168720

38. Dack C, Ross J, Bowers L. The relationship between attitudes towards different containment measures and their usage in a national sample of psychiatric inpatients. J Psychiatr Ment Health Nurs. (2012) 19:577-86. doi: $10.1111 /$ j.1365-2850.2011.01832.x

39. Bowers L, Haglund K, Muir-Cochrane E, Nijman H, Simpson A, Van Der Merwe M. Locked doors: a survey of patients, staff and visitors. J Psychiatr Ment Health Nurs. (2010) 17:873-80. doi: 10.1111/j.1365-2850.2010.01614.x

40. Schweizerische Akademie der Medizinischen Wissenschaften [SAMW]. Zwangsmassnahmen in der Medizin. Medizin-ethische Richtlinien (2017). Available online at: https://www.samw.ch/dam/jcr:b017b872.../richtlinien_ samw_zwangsmassnahmen.pdf

41. Ranieri V, Madigan K, Roche E, Bainbridge E, McGuinness D, Tierney K, et al. Caregivers' perceptions of coercion in psychiatric hospital admission. Psychiatr Res. (2015) 228:380-5. doi: 10.1016/j.psychres.2015.05.079

42. Fugger G, Gleiss A, Baldinger P, Strnad A, Kasper S, Frey R. Psychiatric patients' perception of physical restraint. Acta Psychiatr Scand. (2016) 133:221-31. doi: 10.1111/acps.12501

43. Happell B, Harrow A. Nurses' attitudes to the use of seclusion: a review of the literature. Int J Ment Health Nurs. (2010) 19:162-8. doi: $10.1111 / j .1447-0349.2010 .00669 . x$

44. Jaeger M, Ketteler D, Rabenschlag F, Theodoridou A. Informal coercion in acute inpatient setting-Knowledge and attitudes held by mental health professionals. Psychiatr Res. (2014) 220:1007-11. doi: 10.1016/j.psychres.2014.08.014

45. Soininen P, Valimaki M, Noda T, Puukka P, Korkeila J, Joffe G, et al. Secluded and restrained patients' perceptions of their treatment. Int J Ment Health Nurs. (2013) 22:47-55. doi: 10.1111/j.1447-0349.2012.00838.x

46. Strout TD. Perspectives on the experience of being physically restrained: an integrative review of the qualitative literature. Int J Ment Health Nurs. (2010) 19:416-27. doi: 10.1111/j.1447-0349.2010.00694.x

47. Husemann M, Schulz M, Löhr M. The safewards model in Akutsetting, relieving conflicts-allowing participation. Psych Care (2014) 20:212-20. doi: $10.1055 / \mathrm{s}-0034-1384781$

48. Baker JA, Bowers L, Owiti JA. Wards features associated with high rates of medication refusal by patients: a large multi-centered survey. Gen Hosp Psychiatr. (2009) 31:80-9. doi: 10.1016/j.genhosppsych.2008.09.005

49. Bowers L, Alexander J, Simpson A, Ryan C, Carr-Walker P. Student psychiatric nurses' approval of containment measures: relationship to perception of aggression and attitudes to personality disorder. Int J Nurs Stud. (2007) 44:349-56. doi: 10.1016/j.ijnurstu.2005.03.002

50. Bowers L, Hammond N, James K, Quirk A, Robson D, Stewart D. Characteristics of acute care associated with the presence of a psychiatric intensive care unit, and transfers of patients to it. J Psychiatr Intens Care (2012) 8:66-77. doi: 10.1017/S174264641200012X

51. Bowers L, Van Der Merwe M, Nijman H, Hamilton B, Noorthorn $\mathrm{E}$, Stewart $\mathrm{D}$, et al. The practice of seclusion and time-out on acute psychiatric wards: the City-128 study. Arch Psychiatr Nurs. (2010) 24:275-86. doi: 10.1016/j.apnu.2009.09.003

52. Hottinen A, Valimaki M, Sailas E, Putkonen H, Joffe G, Noda T, et al. Attitudes towards different containment measures: a questionnaire survey in Finnish adolescent psychiatry. J Psychiatr Ment Health Nurs. (2012) 19:521-7. doi: $10.1111 / j .1365-2850.2011 .01820 . x$

53. Jalil R, Huber JW, Sixsmith J, Dickens GL. Mental health nurses' emotions, exposure to patient aggression, attitudes to and use of coercive measures: cross-sectional questionnaire survey. Int J Nurs Stud. (2017) 75:130-8. doi: 10.1016/j.ijnurstu.2017.07.018

54. Muir-Cochrane E, Bowers L, Jeffery D. Comparison in attitudes between nursing and other students in relation to containment measures for 
disturbed psychiatric patients. Nurse Edu Today (2009) 29:83-90. doi: 10.1016/j.nedt.2008.07.002

55. Pettit SA, Bowers L, Tulloch A, Cullen AE, Moylan LB, Sethi F, et al. Acceptability and use of coercive methods across differing service configurations with and without seclusion and/or psychiatric intensive care units. J Adv Nurs. (2017) 73:966-76. doi: 10.1111/jan.13197

56. Elmer T, Rabenschlag F, Schori D, Zuaboni G, Kozel B, Jaeger S, et al. Informal coercion as a neglected form of communication in psychiatric settings in Germany and Switzerland. Psychiatr Res. (2017) 262:400-6. doi: 10.1016/j.psychres.2017.09.014

57. Tingleff EB, Bradley SK, Gildberg FA, Munksgaard G, Hounsgaard L. "Treat me with respect". A systematic review and thematic analysis of psychiatric patients' reported perceptions of the situations associated with the process of coercion. J Psychiatr Ment Health Nurs. (2017) 24:681-98. doi: $10.1111 /$ jpm.12410
58. Meijer E, Schout G, de Jong G, Abma T. Regaining ownership and restoring belongingness: impact of family group conferences in coercive psychiatry. $J$ Adv Nurs. (2017) 73:1862-72. doi: 10.1111/jan.13270

Conflict of Interest Statement: The authors declare that the research was conducted in the absence of any commercial or financial relationships that could be construed as a potential conflict of interest.

Copyright (C) 2018 Reisch, Beeri, Klein, Meier, Pfeifer, Buehler, Hotzy and Jaeger. This is an open-access article distributed under the terms of the Creative Commons Attribution License (CC BY). The use, distribution or reproduction in other forums is permitted, provided the original author(s) and the copyright owner(s) are credited and that the original publication in this journal is cited, in accordance with accepted academic practice. No use, distribution or reproduction is permitted which does not comply with these terms. 